\begin{tabular}{|l|l|}
\hline Postprint Version & 1.0 \\
\hline Journal website & http://www.sciencedirect.com/science/article/pii/S0266613813002489 \\
\hline Pubmed link & http://www.ncbi.nlm.nih.gov/pubmed/24074733 \\
\hline DOI & $10.1016 /$ j.midw.2013.08.010 \\
\hline
\end{tabular}

This is a NIVEL certified Post Print, more info at http://www.nivel.eu

\title{
Work and workload of Dutch primary care midwives in 2010
}

\author{
T.A. WIEGERS ${ }^{*}$, J.C. WARMELINK ${ }^{* * \#}$, E.R. SPELTEN ${ }^{\#}$, G.M.T. KLOMP ${ }^{\#}$, E.K. HUTTON ${ }^{\#}$ \\ \# Department of Midwifery Science, AVAG and the EMGO Institute for Health and Care \\ Research, VU University Medical Center, Amsterdam, the Netherlands; \\ ${ }^{\$}$ Faculty of Health Sciences, McMaster University, Hamilton, Canada
}

\begin{abstract}
Objective: To re-assess the work and workload of primary care midwives in the Netherlands.

Background: In the Netherlands most midwives work in primary care as independent practitioners in a midwifery practice with two or more colleagues. Each practice provides 24/7 care coverage through office hours and on-call hours of the midwives. In 2006 the results of a time registration project of primary care midwives were published as part of a 4-year monitor study. This time registration project was repeated, albeit on a smaller scale, in 2010.

Method: As part of a larger study (the Deliver study) all midwives working in 20 midwifery practices kept a time register 24 hours a day, for one week. They also filled out questionnaires about their background, work schedules and experiences of workload. A second component of this study collected data from all midwifery practices in the Netherlands and included questions about practice size (number of midwives and number of clients in the previous year).

Findings: In 2010, primary care midwives actually worked on average 32.6 hours per week and approximately $67 \%$ of their working time (almost 22 hours per week) was spent on client-related activities. On average a midwife was oncall for 39 hours a week and almost 13 of the 32.6 hours of work took place during on-call-hours. This means that the total hours that an average midwife was involved in her work (either actually working or on-call) was almost 59 hours a week. Compared to 2004 the number of hours an average midwife was actually working increased by 4 hours (from 29 to 32.6 hours) while the total number of hours an average midwife was involved with her work decreased by 6 hours (from 65 to 59 hours). In 2010, compared to 2001-2004, the midwives spent proportionally less time on direct client care (67\% vs. $73 \%$ ), although in actual number of hours this did not change much (22 vs. 21). In 2009 the
\end{abstract}


average workload of a midwife was 99 clients at booking, 56 at the start of labour, 33 at childbirth, and 90 clients in postpartum care.

Conclusion: The midwives worked on average more hours in 2010 than they did in 2004 or 2001, but spent these extra hours increasingly on non-clientrelated activities.

\section{INTRODUCTION}

In the Netherlands maternity care is provided by midwives, gynaecologists and some family doctors (general practitioners), and home birth is still a viable option for women with uncomplicated pregnancies. In recent years the debate about the Dutch system of maternity care has re-emerged following the EURO-PERISTAT data on perinatal mortality in Europe and the unfavourable position of the Netherlands in those data (EURO-PERISTAT 2008). The current debate focuses on ways to reduce the perinatal death rate by improving the quality of care. Central elements in this debate are, as formulated by the Steering committee Pregnancy and Childbirth (2009): client-centred care: mother and (unborn) child should always be the focus of the care provision; continuity of labour care: no labouring woman should be left alone; cooperation between care providers: joint responsibility for the optimal care for mother and child. Since the release of the Steering committee advice all midwifery practices are expected to participate in a local maternity care consultation and cooperation group (in Dutch: Verloskundig SamenwerkingsVerband or: VSV) with the local hospital. In 2004 only $42 \%$ of midwifery practices participated in a VSV. In Box 1 a short overview is given of the position of midwives in the health care system in the Netherlands

\section{[Box 1]}

To be able to provide optimal care it is important that the workload of care providers is not too high.

The Dutch Healthcare Authority (NZa), the healthcare market supervisor, plays an indirect role in defining the workload of independent care providers, by deciding on the maximum fees they are allowed to ask for their services and thus regulating their income. For midwives the NZa also decides how many 'care units' a full-time working midwife is expected to see, thereby indirectly setting the norm for an acceptable workload. In Box 2 a short explanation is given about workload in Dutch midwifery care, and the definition of a 'care unit'.

\section{[Box 2]}

In this paper we present data about the work and workload of primary care midwives in the Netherlands in 2010 and we compare these with data from 2001 and 2004 from an earlier study (Wiegers, 2006). The work of midwives is described in terms of client- and non-client-related activities and face-to-face client care is subdivided in different aspects: care during pregnancy, during labour and during the postpartum period. Workload of midwives is described in two ways: first as an estimate of an 
Wiegers, T.A., Warmelink, J.C., Spelten, E.R., Klomp, G.M.T., Hutton, E.K. Work and workload of Dutch primary care midwives in 2010. Midwifery: 2014, 30(9), 991-997

average case-load, by calculating the number of women in the different stages of maternity care per midwife. Second, by calculating the average number of hours a midwife is working, including and excluding the on-call hours.

\section{WORKLOAD OF MIDWIVES IN INTERNATIONAL PERSPECTIVE}

There is almost no comparable information about working hours of (independent) midwives from other countries. However, in an Australian study by Paterson et. al. the average working hours of midwives was found to be 28.7 hours per week (Paterson et al. 2010).

Data about the average time midwives spend with their clients is equally scarce, but there are 'old' recommendations, for instance in the 'framework for workforce planning and decision-making for midwifery services': Birthrate Plus (Ball \& Washbrook 1996). Their formula for assessing midwife hours per client for caseload based work includes 9 hours for antenatal care, 17 hours for intrapartum care (including some allowance for a second midwife to attend a home birth) and between 10 and 15 hours for postnatal care, adding up to 38 hours in case of normal/low risk pregnancy and birth and 43 hours for complicated/high risk pregnancy and birth. A recent study from New Zealand found that with first-time mothers giving birth at home or in hospital, midwives were on average present for 8.7 hours at home births and 10.1 hours at hospital births (Miller \& Skinner, 2012).

As is presented in Box 2, the fees of independent midwives in the Netherlands are based on a caseload of 105 'care units' per year. In Australia several recent studies mention a personal caseload of 40 to 45 women per year for a full-time working midwife (Williams et al. 2010; Forster et al. 2011; Toohill et al. 2012). An older study from the UK also mentions a caseload of around 40 per year (Benjamin et al. 2001) but more recent recommendations are 25 to 28 births a year. As the report says: 'The recommended total care ratios indicate the maximum number of women that a midwife can provide antenatal, intrapartum and postnatal care for within the service', that is, apart from her other roles 'such as practice development, audit and risk management, breastfeeding adviser, antenatal screening coordinators and so on' (RCoA, RCM, RCOG, RCPCH, 2007).

\section{BACKGROUND DATA ON PRIMARY CARE MIDWIFERY IN THE NETHERLANDS}

There were 2.612 midwives working in the Netherlands on January 1, 2011 with the majority (73\%) working in primary care. A growing number (27\% in 2011, compared to $16 \%$ in 2001) are employed by hospitals and work under supervision of a gynaecologist, where they care for women who have been referred to secondary care. These hospital midwives are not included in this study.

Over the last decade the number of practising midwives has increased. In January 2011 there were 1,599 midwives practicing in primary care (excluding 311 locums), that is on average 6 midwives per 10,000 women of childbearing age (15-39 years). In 2004 that was 5.3 and in 2000 that was 4.3 midwives per 10,000 women of childbearing age. Primary care midwives work in private practice, either as selfemployed practitioner, alone or in partnership with one or more other midwives, or as employee in someone else's practice or as locum, filling in vacancies in midwifery practices on a temporary basis. Their average number of working hours 
per week in 2010 was estimated to be 25.7 hours (Hingstman \& Kenens 2010). The number of midwifery practices has increased from 452 in 2004 to 519 in 2011 (Kenens \& Hingstman 2004, 2011). The average practice size has remained fairly stable over the years and is 3.1 midwives per practice in 2011. Standard client care by primary care midwives is considered to consist of a booking visit, on average eleven prenatal consultations, one consultation including ultrasound, care during labour and birth, five or six postnatal visits and a final contact six week post-partum. Most clients will receive more than one ultrasound, but the majority of those ultrasounds will not be performed by the client's own midwife, at her own practice. Therefore, only one ultrasound per client is included in the calculation for standard client care. During labour a midwife will visit a client several times to check on her progress, but will only stay with her when she deems the birth to be imminent or when the woman specifically asks her to stay. Referral will have an impact on the average time spend with a client during labour and birth, because often the midwife will not stay with the woman after having handed over care to the gynaecologist or hospital midwife.

\section{RESEARCH QUESTIONS}

The work of primary care midwives was publicly debated around the turn of the century, when because of shortages in all sectors of maternity care the workload was high. Research related to midwifery shortages of 10 to 15 years ago, based on data from 2000-2004 is reported in earlier work (Wiegers, 2006). Even before that time workload of midwives was an issue as is reflected in earlier research dating from 1993 and 1996 (Jabaaij et al. 1994; Jabaaij \& Meijer 1996). The data presented here are the result of a replication, albeit on a smaller scale, of the more recent research. The research questions are: 1 . What is the workload of a primary care midwife in the Netherlands, in terms of duration: the number of hours a primary care midwife is working per week, and in terms of caseload: the number of clients a primary care midwife sees in a year? 2. What is the content of the work of a primary care midwife in the Netherlands, in terms of: time spend on client and non-client related activities, and in terms of time spend on different aspects of client care? By comparing the results of this research with those of the earlier research an indication can be given of the changes in workload and time spent on client care and non-client related activities over the years 2001 to 2010 .

\section{PARTICIPANTS AND METHODS}

This study is part of a larger project, the 'Deliver-Study', of which background and design are described extensively in another publication. The Deliver study is a multicentre prospective dynamic cohort study aimed at evaluating the quality, organisation and accessibility of primary midwifery care in the Netherlands (Manniën et al. 2012). In short: a sample of 20 midwifery practices were included in the study to collect midwifery and client data. Selection was based on three criteria: region, level of urbanisation and practice type (duo- or group-practice). Practices that declined cooperation were replaced with comparable practices in the same region. During one year a lot of data were collected in these Deliver practices, including client medical records and client surveys, but relevant for this paper is that 
in the spring of 2010 all midwives working in the Deliver practices kept a detailed registration of all their work-related activities with an accurate timetable, covering 24 hours a day during one full week (Monday to Monday). Midwives were asked to note their time of arrival at the practice, the start and finish time of the scheduled appointments with pregnant women, the number, duration and purpose of all scheduled or unscheduled consultations, the number and duration of postpartum home visits and throughout the day their travelling time. When called to a birth they were asked to note the time (and duration) of the call, the time they spent with the woman, and their travelling time. They were also asked to note the time spent on administrative tasks, whether those tasks were client-related or not, the time spent at meetings with colleagues, other health care professionals, or any others, and the start and duration of their on-call hours. In addition they were asked to rate their work pressure each workday on a visual-analogue-scale of $10.0 \mathrm{~cm}$. In other words, the midwives were asked to provide a complete description including a timetable of their work: what they did, where they went, how long it took, how the work was spread out over twenty-four-hours and over weekdays and weekend and how they experienced it. The use of self-reported data was chosen, because in earlier studies no difference was found when self-reported data were compared to observational data (Jabaaij et al. 1994).

In an additional questionnaire the individual midwives in these 20 Deliver practices provided information about their background, their work experience, their average workload, and their professional attitudes.

In addition to the data collection from individual midwives in the 20 Deliver practices, in a national practice survey a questionnaire was send to all 519 midwifery practices in the Netherlands, including the Deliver practices, with questions about the organisation of their practice, the number of midwives working there, the number of bookings, births and postpartum clients in the previous year (2009), the average number of on-call hours for each midwife, and the working relation with other care providers, including participation in the local VSV.

\section{RESULTS}

In the 20 Deliver practices a total of 108 midwives and 28 practice assistants participated in the time registration (100\% response rate), 99 of the 108 midwives returned the individual questionnaire ( $91.7 \%$ response rate). Registration data from the practice assistants are not reported here.

Of the 519 midwifery practices in the national survey 319 returned a (mostly) completed practice questionnaire (61.5\% response rate). A total of $75 \%$ of these practices participated in a local VSV, compared to 42\% in 2004.

Background data about the 99 midwives in the Deliver practices are presented in Table 1, alongside data from the midwifery occupational registration (Hingstman and Kenens 2010) which includes all midwives practicing in primary care in 2010. The 20 Deliver practices were relatively large, with an average of 5.4 midwives per practice, compared to an average of 4.2 in the 319 national survey practices. Of the 319 national survey practices 209 (65.5\%) also provided data about the number of clients in the previous year (see Table 2). The Table shows that the average number of bookings in 2009 was 416 per practice, with an average of 237 clients starting labour in primary care assisted by their midwife and an average of 
140 women giving birth in primary care. Of the women who gave birth in primary care on average $64 \%$ gave birth at home. An average of 367 women received postpartum care from the midwife. These data tell us that in 2009 an average midwife had 99 bookings, was the responsible care provider for 56 women at the start of labour, was responsible for 33 births, of which 21 at home, and for 90 postpartum women. In essence, this could be regarded as the average case-load of a Dutch primary care midwife in 2009. The results from the time registration in the 20 Deliver practices show that primary care midwives in this study worked on average 32.6 hours per week and approximately $67 \%$ of their time ( 21.8 hours per week) was spend on client-related activities (see Table 3). The average number of working hours per week increased since 2001. An average of $13 \%$ of the actual work (4.3 hours per week) took place during evenings or nights, that is: between $7.00 \mathrm{PM}$ and 7.00 AM (not in table). The increase in total working time is almost exclusively due to an increase in non-client related activities.

he average of 32.6 working hours per midwife per week is calculated over all 20 Deliver practices.

The differences between these practices range from 21.8 to 44.2 working hours per midwife per week.

But there are much larger differences between individual midwives during the registration week, ranging from less than ten to more than fifty hours (see Figure 1). This is related to the fact that most midwives are self-employed and do not have fixed working hours. They can alternate busy periods with quieter periods, for instance by having a few days off or working less hours after having been on call. Compared to the results from 2004 and 2001 more midwives were working 50 hours a week or more in 2010.

The average of 32.6 hours per week in 2010 is the number of hours the midwife has actually been working. It does not include the on-call hours in which no work was done. However, being on call can be regarded as work in a sense that it means: staying in the area, staying alert and sober, being able and prepared to drop whatever you are doing to respond to a call and visit a client if needed. On average a midwife was on call during 39 hours a week, and working during 12.7 of those 39 hours. The other 19.9 hours were scheduled working hours (see Figure 2). This means that an average midwife was involved in her work during 58.9 hours a week, of which she was 32.6 hours actually working.

The change over the years is gradual, and the most striking difference with earlier results is the shift in the hours between scheduled work and work during on-call hours (see Table 4). The total number of on-call hours per midwife has been reduced from 56.4 hours per week in 2001 to 39.0 hours per week in 2010. Work during these on-call hours decreased in the actual number of hours but not in percentage, from 17.7 to 12.7 hours per week, but still about $32 \%$ of the total on-call hours, while scheduled work hours as a proportion of all work hours increased from $38.6 \%$ in 2001, via $40.8 \%$ in 2004 to $61.0 \%$ in 2010 .

Based on the time-registrations during one week by 108 midwives in 20 Deliver practices the total time a midwife will spend on a 'standard care' client (a woman at low risk at the onset of labour), from the booking visit until the final contact six week post-partum, is calculated (see Table 5).

It is important to note that this was not a longitudinal study, so no actual clients were followed to measure the time midwives spend with them. The calculations are based 
on the midwifery time registrations and represent the time midwives have spent on different client contacts during one week.

These data are then combined with standard client care to calculate an average of time spent with a client throughout pregnancy, labour, childbirth and the postpartum period. Because about $41 \%$ of all clients who receive care from a midwife at the start of labour will be referred to secondary care (see Table 2), time spent on care during labour and birth in this calculation does not represent the exemplary low-risk, uncomplicated childbirth (the 'care unit' as mentioned in Box 2), but relates to a mixture of not-referred and referred clients.

In 2010 the total (calculated) time for standard care per client is found to be 732 minutes (12.2 hours) which is an increase compared to earlier years. In 2001 all aspects of standard care together added up to a total of 601 minutes, that is 10 hours of direct client care per 'standard care' client. In 2004 that was increased to 712 minutes, or almost 12 hours per client. The data show that the time spent on all separate aspects of direct client care has increased, except the time attending labour and birth. This may be related to the high referral rate, reducing the average time spent with a birthing woman, because of the referral and hand-over to secondary care.

The reduction of the fulltime caseload (see Box 2) and the increased time spent on direct client care has led to a reduced overall work pressure as measured with a daily recording on a visual-analoguescale.

On that $10.0 \mathrm{~cm}$ long scale the overall average work pressure (measured in $\mathrm{cm}$ ) was 4.27 in 2001, 4.86 in 2004 and 4.29 in 2010 (see Table 6). In all the years (20012004 and 2010) the recorded work pressure was significantly $(\mathrm{p}<0.01)$ correlated to the number of hours the midwives had worked.

\section{DisCUSSION}

The 'standard caseload' of a Dutch midwife (the number of clients a full-time working midwife will need to see, to reach the 'normative' annual income) has been reduced from 120 per year in 2001 to 105 per year in 2010. One of the reasons behind this reduction has been to give midwives the opportunity to spend more time with each client. This study has shown that midwives indeed spend more time with their clients, although the proportion of time devoted to non-client-related activities has increased much more. This may be the result of the increased emphasis on interdisciplinary cooperation in order to reduce perinatal mortality and morbidity and improve the quality of maternity care overall. In 2004 only $42 \%$ of practices participated in a local VSV, in 2010 that was increased to approximately $75 \%$. The average of 32.6 working hours per week in 2010 is higher than was found in the earlier years 2004 and 2001, and is also higher than the average of 25.7 calculated for all independent primary care midwives in the NIVEL occupational registration of midwives in 2010 (Hingstman \& Kenens 2010).

However, those figures are not based on a time registration as are ours, but on questionnaires and midwives may have underestimated their actual working hours because these are irregularly distributed over a day or a week. It is difficult to make an international comparison, because information about working hours of (independent) midwives is scarce, but in an Australian study by Paterson et. al. the 
average working hours of midwives was less than in our study: 28.7 hours per week (Paterson et al. 2010).

The average time spent with a standard client is found to be 12.2 hours, of which 4.6 hours during pregnancy, 3.5 hours during labour and birth and 4.1 hours in the postnatal period. The time spent during labour and birth in our study is much less than what was found in the New Zealand study where at home births midwives were on average present for 8.7 hours and in hospital for 10.1 hours (Miller \& Skinner, 2012). However, it is important to remember that in the New Zealand study midwives stayed with their clients even when referral was required. Comparing our results with formula from Birthrate Plus (Ball \& Washbrook 1996) leads to the conclusion that, if, as in our study, one third of the midwife's time is spent on nonclient-related activities, then 25 to 29 hours are spent with an average client. That is more than twice as much as in the Netherlands.

Comparison at the level of 'case load'(105 'care units' (see Box 2)) in the Netherlands, 40-45 clients in Australia and 25-28 clients in the UK) also shows that the differences between countries are large.

Because some of the recent developments, such as lowering the 'standard caseload' and increasing emphasis on continuity of care, were expected to lead to an increase in the time a midwife spends with a woman during labour and birth, the reduction we found in the average time a midwife spends attending a birthing woman needs explaining. It could be that other developments, occurring simultaneously, lead to a decrease of a midwife's time with a birthing woman. For instance the increasing referral rate and the reduced length of the on-call period may provide some explanation. In 2004 the national referral rate to secondary care for women starting labour with a midwife in primary care was 26.7\% (PRN, 2007), in 2008 that rate was $31.7 \%$. The 209 practices included in the national survey of this study reported a referral rate of approximately $41 \%$ in 2009. Many referrals take place in the early stages of labour, either because progression is slow, or because of the need for pain relief.

Depending on their workload, midwives will often leave their client after handing care over to the hospital staff, especially since there are more midwives on the hospital staff, thus reducing the time the primary care midwife spends with the labouring woman.

In addition, as a consequence of the fact that it was the midwife registering her time with a woman and not the woman registering when a midwife was present, a handover to a colleague meant a 'new' case in the data set. Because of the reduced length of the average on-call period, a midwife will more frequently hand over a client to a colleague, thus reducing the time she personally is attending this particular birth. Moreover, due to the short period of registration a relatively large number of births may have only been partially registered, because they started before or lasted until after the registration period. This means that the study design itself may have contributed to these results.

Seventy of the 108 midwives in the study together attended 123 births during one week, for 53 of which the time of birth of a baby was registered on the time registration form, indicating that the midwife was present during the birth of the baby. In the other 70 cases the woman may have been referred to secondary care, or to a colleague, or the baby was born after the registration period was finished, so the time of birth could not be included in the registration. If the intrapartum referral 
rate during the time registration was the same as the year before, $41 \%$, then that would have led to 50 referrals, leaving 20 births to be incompletely registered because of hand-over to a colleague or partly occurring outside the registration period.

It is not possible to calculate the average number of 'care units' per midwife from these data, because we lack information about the timing of the referrals during pregnancy. Early referrals add less to a 'care unit' than late referrals. However, we can make an estimation: the average number of 'care units' per midwife will be less than 99 (the number of bookings per midwife) and more than 56 (the number of births per midwife starting in primary care). If the number of women receiving postpartum care from a midwife can be regarded as an indication of the number of women receiving also the majority of their prenatal care from a midwife, then the number of 'care units' can be estimated to be around 87 per midwife.

To take this a step further, should we regard the 732 minutes per standard client care to be an indication of the average time given to a 'care unit', than we can calculate that a midwife in this study cared for 1.8 'care units' per week, what amounts to 92 'care units' a year, or 87 'care units' over a period of 49 weeks (a year minus a holiday). However, this is speculation based on calculation and more research is needed to understand the real workload of primary care midwives in the Netherlands.

\section{LIMITATIONS}

This study has some limitations. Firstly, the use of self-reported data is not undisputed, because it is impossible to know the accuracy with which the midwives have filled out their time table. However, in earlier studies no difference was found when self-reported data were compared to observational data (Jabaaij et al. 1994). Another limitation that was already mentioned is the length of the time registration period in this study: one week. This has made it difficult to make an accurate estimate of the average time a midwife spends with a woman during childbirth, because every labour and birth in progress during the start or the end of the registration period has been only partly visible in the research data. And with just one week of registration this obviously happens relatively more often than with a registration of a longer duration. On the other hand, the short period made it for the midwives easier to comply, which will have improved the accuracy of the time registration in general. Finally, it is important to realise that an accurate time registration, especially when performing such diverse tasks as those of a midwife, tends to leave out the quieter moments, because they are often not seen as work. That means, that the average of 32.6 hours for independently working midwives, might be equivalent to a full-time 36-hours salaried job. But we have no way of knowing that for sure from these data.

\section{CONCLUSION}

In 2010 Dutch midwives worked on average 32.6 hours a week of which two thirds was directly client-related and one third was spend on non-client-related activities such as practice and financial administration and organisation, meetings, study and other activities. The majority of all the work $(19.9$ hours $=61 \%)$ took place during scheduled hours. On average midwives were on call for 39 hours per week in this 
registration period. The conclusion is that the average professional involvement of a midwife was 58.9 hours per week. The increase in total working time since 2004 is almost exclusively due to an increase in non-client related activities. The average time spent on standard client care has increased slightly on all aspects, except on attending labour and birth. The overall conclusion is that midwives worked on average more hours in 2010 than in 2004 or 2001, but they spend an increasing proportion of their time on non-client-related activities.

\section{REFERENCE LIST}

Ball J.A., Washbrook M. (1996) Birthrate Plus. A framework for workforce planning and decision-making for midwifery services. Elsevier Science Limited. Edinburgh

Benjamin Y., Walsh D., Taub N. (2001) A comparison of partnership caseload midwifery care with conventional team midwifery care: labour and birth outcomes. Midwifery 17: 234-240

EURO-PERISTAT, SCPE, EUROCAT, EURONEOSTAT (2008). European Perinatal Health Report. 2008. www.europeristat.com

Forster D., Newton M., McLachlan H.L., Willis K. (2011) Exploring implementation and sustainability of models of care: can theory help? BMC Public Health 11 (Suppl 5):58

Hingstman L., Kenens RJ. (2010) Cijfers uit de registratie van verloskundigen. Peiling 2010. [Data from the registration of midwives 2010] Utrecht, NIVEL

Hingstman L., Kenens RJ. (2011) Cijfers uit de registratie van verloskundigen. Peiling 2011. [Data from the registration of midwives 2011] Utrecht, NIVEL

Jabaaij L., Winckers M., Hingstman L., Meijer W. (1994) De vrijgevestigde verloskundige in Nederland: werk en werkdruk. [The self-employed midwife in the Netherlands: work and working pressure] Utrecht, NIVEL

Jabaaij L. Meijer W. (1996) Home births in the Netherlands: midwifery related factors of influence. Midwifery 12: 129-135

Kenens RJ., Hingstman L. (2004) Cijfers uit de registratie van verloskundigen. Peiling 2004. [Data from the registration of midwives 2004] Utrecht, NIVEL

Manniën J., Klomp T., Wiegers T., Pereboom M., Brug J., Jonge A. de, Meijde van der M., Hutton E., Schellevis F., Spelten E. (2012) Evaluation of primary care midwifery in the Netherlands: design and rationale of a dynamic cohort study (DELIVER). BMC Health Services Research, 12:69

Miller S., Skinner J. (2012) Are first-time mothers who plan home birth more likely to receive evidence-based care? A comparative study of home and hospital care provided by the same midwives. Birth 39 (2): 135-144

Paterson J.L., Dorrian J., Pincombe J., Grech C., Dawson D. (2010) Mood change and perception of workload in Australian midwives. Industrial Health 48: 381-389 Royal College of Anaesthetists,

Royal College of Midwives, Royal College of Obstetricians and Gynaecologists, Royal College of Paediatrics and Child Health (2007) SAFER CHILDBIRTH: Minimum Standards for the Organisation and Delivery of Care in Labour. RCOG Press London http://www.rcog.org.uk/files/rcog-corp/uploaded-files/WPRSaferChildbirthReport2007.pdf Stichting Perinatale Registratie Nederland (2007) Perinatale zorg in Nederland 2004.

[Perinatal care in the Netherlands 2004] Utrecht, PRN

Stichting Perinatale Registratie Nederland (2011) Perinatale zorg in Nederland 2008.

[Perinatal care in the Netherlands 2008] Utrecht, PRN

Stuurgroep zwangerschap en geboorte (2009) Een goed begin. Veilige zorg rond zwangerschap en geboorte, [Steering group Pregnancy and childbirth: A safe beginning. Safety in care concerning pregnancy and childbirth] Utrecht

Toohill J., Turkstra E., Gamble J., Scuffham P.A. (2012) A non-randomised trial investigating the costeffectiveness of Midwifery Group Practice compared with standard maternity care arrangements in one Australian hospital. Midwifery 28: e874-e879

Wiegers TA (2006) Workload of primary care midwives. Midwifery 23 (4): 425-432

Williams K, Lago L, Lainchbury A, Eagar K. (2010) Mothers' views of caseload midwifery and the value of continuity of care at an Australian regional hospital. Midwifery;26(6):615-21. 
Wiegers, T.A., Warmelink, J.C., Spelten, E.R., Klomp, G.M.T., Hutton, E.K. Work and workload of Dutch primary care midwives in 2010. Midwifery: 2014, 30(9), 991-997

\section{TABLES}

Box 1. Midwives in Dutch health care

In the Netherlands midwives are medical practitioners with competencies restricted to independently provide care during 'normal' pregnancy, childbirth and the postpartum period. Midwifery education is a 4-year vocational training (at a university for applied science) with a degree at bachelor level. Women with an uncomplicated pregnancy are expected to receive care from a primary care midwife and are not required to consult a gynaecologist/obstetrician, the midwife is fully responsible for the care provided. But when complications arise, or threaten, or the woman requests a form of pain relief that can only be given in secondary care, the midwife will have to transfer responsibility by referring the woman to a gynaecologist. She can choose to stay with her client or leave her in the care of a hospital midwife or nurse, but from the moment of referral onwards the gynaecologist is responsible for the care provided.

Box 2. workload 'norms' or 'standard caseload'

There are no formal workload 'norms' for primary care midwives, because most are self-employed, meaning they can decide for themselves how many clients they want to accept and how much time they want to spend with each client. That means there is no workable definition for fulltime or part-time working in midwifery care in the Netherlands. However, there is a 'standard caseload' used for calculating the fees for service, that is: the number of 'care units' a full-time working midwife will need to see, to reach the 'normative' annual income. A 'care unit' is the equivalent of one client receiving care throughout pregnancy, childbirth and the postpartum period. This 'standard caseload' has decreased from 120 'care units' per year in 2001 to 105 per year in 2010. 
Wiegers, T.A., Warmelink, J.C., Spelten, E.R., Klomp, G.M.T., Hutton, E.K. Work and workload of Dutch primary care midwives in 2010. Midwifery: 2014, 30(9), 991-997

\begin{tabular}{|c|c|c|c|c|}
\hline \multicolumn{4}{|c|}{ Midwives in Deliver practices } & \multirow{2}{*}{$\begin{array}{l}\text { Primary care midwives in the Netherlands } \\
\%\end{array}$} \\
\hline & Mean (sd) & $N$ & $\%$ & \\
\hline Age & $38.0(10.9)$ & & & \\
\hline$-20-29$ & & 28 & 28.3 & 30.3 \\
\hline$-30-39$ & & 28 & 28.3 & 33.2 \\
\hline$-40-49$ & & 24 & 24.2 & 20.9 \\
\hline$-50-59$ & & 16 & 16.2 & 12.2 \\
\hline$\rightarrow 59$ & & 2 & 2.0 & 1.9 \\
\hline - missing & & 1 & 1.0 & 1.5 \\
\hline \multicolumn{5}{|l|}{ Function } \\
\hline - self-employed & & 72 & 72.7 & 73.5 \\
\hline - employee & & 18 & 18.2 & 11.9 \\
\hline - locum & & 9 & 9.1 & 14.6 \\
\hline Average years working & $12.9(10.0)$ & & & \\
\hline \multicolumn{5}{|l|}{ Practice type } \\
\hline - solo practice & & 0 & 0.0 & 4.9 \\
\hline - duo-practice & & 4 & 4.0 & 13.9 \\
\hline - group practice & & 95 & 96.0 & 81.2 \\
\hline
\end{tabular}

\begin{tabular}{|c|c|c|c|c|}
\hline & Average $N$ & $\%$ of $\mathrm{A}$ & $\%$ of B & $\%$ of $\mathrm{C}$ \\
\hline A: bookings in midwifery practice & 416 & $100 \%$ & & \\
\hline per midwife & 99 & & & \\
\hline$\rightarrow$ referral to secondary care during pregnancy & 179 & $43 \%$ & & \\
\hline per midwife & 43 & & & \\
\hline B: start labour with midwife & 237 & $57 \%$ & $100 \%$ & \\
\hline per midwife & 56 & & & \\
\hline$\rightarrow$ referral to secondary care during labour & 97 & $23 \%$ & $41 \%$ & \\
\hline per midwife & 23 & & & \\
\hline C: give birth in primary care & 140 & $34 \%$ & $59 \%$ & $100 \%$ \\
\hline per midwife & 33 & & & \\
\hline$\rightarrow$ home birth & 90 & $22 \%$ & $38 \%$ & $64 \%$ \\
\hline per miduife & 21 & & & \\
\hline D: receive postpartum care & 376 & $90 \%$ & $159 \%$ & $269 \%$ \\
\hline per midwife & 90 & & & \\
\hline
\end{tabular}


Wiegers, T.A., Warmelink, J.C., Spelten, E.R., Klomp, G.M.T., Hutton, E.K. Work and workload of Dutch primary care midwives in 2010. Midwifery: 2014, 30(9), 991-997
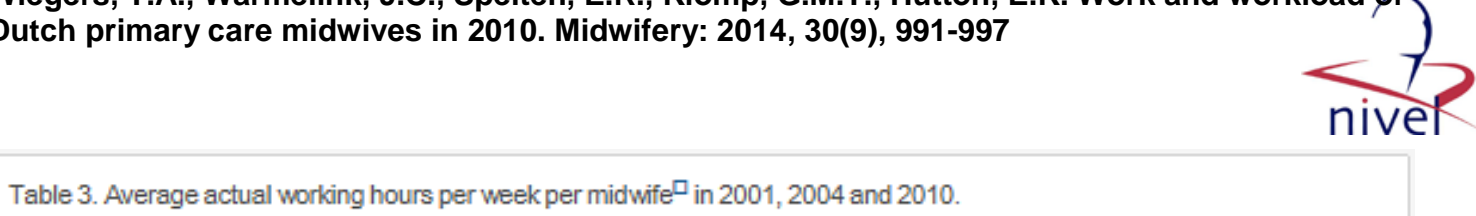

$\square$ being on-call but not actually working is not included in the working hours

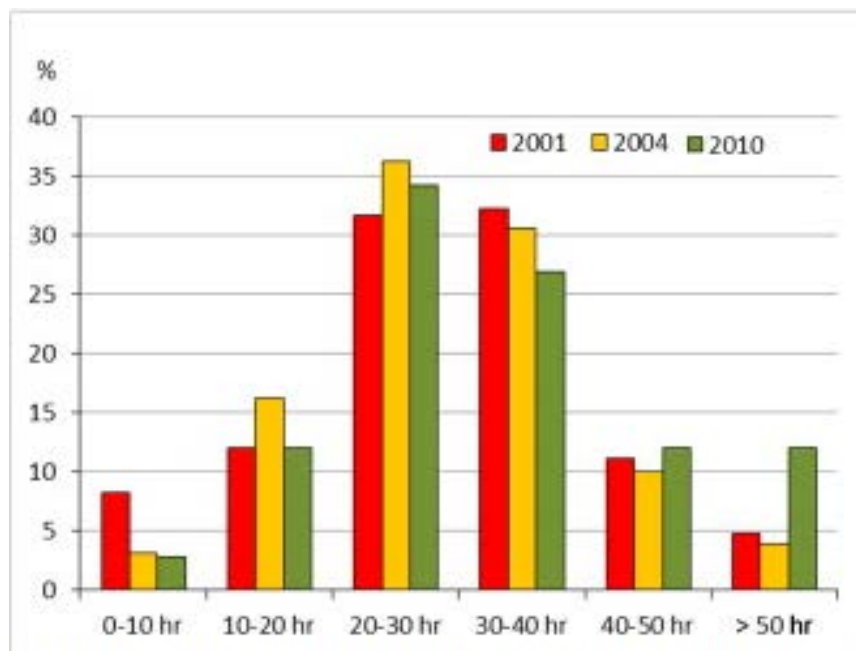

Fig. 1. Range in actual working hours of primary care midwives in 2001, 2004 and 2010.

\section{Overlap between on-call-hours and working hours of primary care midwives in} 2010

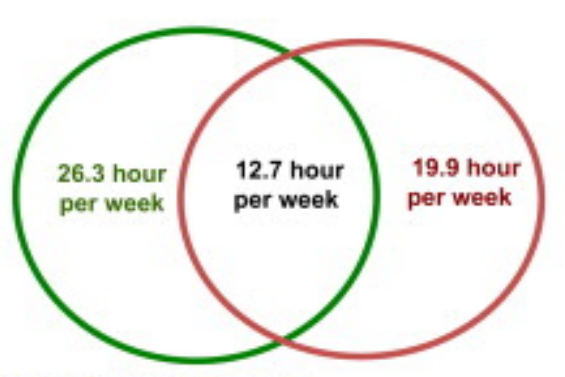

2010 Total on-call-hours: 39.0 per week

Total working hours: 32.6 per week

Total availability: 58.9 hours per week

Fig. 2. Overlap between on-call hours and working hours of primary care midwives in 2010. 
Wiegers, T.A., Warmelink, J.C., Spelten, E.R., Klomp, G.M.T., Hutton, E.K. Work and workload of Dutch primary care midwives in 2010. Midwifery: 2014, 30(9), 991-997

Table 4. Overlap of work and on-call hours per midwife (average) in 2001, 2004 and 2010.

\begin{tabular}{|l|l|l|l|}
\hline work and on-call hours & 2001 (hours) & 2004 (hours) & $\mathbf{2 0 1 0}$ (hours) \\
\hline A. Work during on-call hours & 17.7 & 17.7 & $\mathbf{1 2 . 7}$ \\
\hline B. Work outside on-call hours & 11.2 & 12.2 & $\mathbf{1 9 . 9}$ \\
\hline C. Total working hours (A+B) & 28.9 & 29.9 & $\mathbf{3 2 . 6}$ \\
\hline D. On-call hours & 56.4 & 50.8 & $\mathbf{3 9 . 0}$ \\
\hline E. On-call without actual work (D - A) & 38.7 & 33.1 & $\mathbf{2 6 . 3}$ \\
\hline F. Total 'involved in work' (work+on-call) (C+E) & 68.3 & 63.7 & $\mathbf{5 8 . 9}$ \\
\hline
\end{tabular}

Table 5. Average time (in minutes) per 'standard care' client, in 2001, 2004 and 2010.

\begin{tabular}{|c|c|c|c|}
\hline & 2001 & 2004 & 2010 \\
\hline \multicolumn{4}{|l|}{ aspects of care: } \\
\hline booking visit & 32.9 & 36.3 & 43.1 \\
\hline prenatal consults (11x) & 150.7 & 170.5 & 212.3 \\
\hline consult+ultrasound & 17.5 & 19.9 & 22.1 \\
\hline consult 6 weeks after birth & 16.4 & 16.9 & 24.5 \\
\hline Total client contact at the practice (minutes) & 217.5 & 243.6 & 302.0 \\
\hline birth (including travel time, including referred cases) & 192.7 & 278.5 & 209.0 \\
\hline postnatal visits (incl. travel time) & 190.8 & 189.5 & 221.0 \\
\hline Total & 601.0 & 711.6 & 732.0 \\
\hline
\end{tabular}

Table 6. Correlation between working hours and work pressure in 2001, 2004 and 2010.

\begin{tabular}{|l|l|l|l|}
\hline & 2001 & 2004 & $\mathbf{2 0 1 0}$ \\
\hline Number of midwives & 208 & 229 & $\mathbf{1 0 8}$ \\
\hline Average working hours & $28.9 \mathbf{~ h}$ & $29.9 \mathrm{~h}$ & $\mathbf{3 2 . 6} \mathbf{h}$ \\
\hline Average work pressure (VAS $10.0 \mathrm{~cm})$ & 4,27 & 4,87 & $\mathbf{4 , 2 9}$ \\
\hline Pearson Correlation & $0.306^{\mathrm{Da}}$ & $0.320^{\mathrm{Da}}$ & $\mathbf{0 . 4 2 2 ^ { \square \square }}$ \\
\hline
\end{tabular}

DCorrelation is significant at the 0.01 level (2-tailed). 